\title{
Weniger Exazerbationen mit pflanzlicher Zusatztherapie
}

\begin{abstract}
- Cineol ist Bestandteil von Eukalyptusöl und wirkt antientzündlich und antioxidativ. Das Cineolpräparat Soledum ${ }^{\circ}$ forte ist nicht nur zur Behandlung von banalen Erkältungskrankheiten zugelassen, sondern auch als Komedikation zur Therapie chronisch entzündlicher Atemwegserkrankungen.
\end{abstract}

Zur Wirkung von Cineol stellte Prof. Dr. Heinrich Worth, Klinikum Fürth, die Ergebnisse einer eigenen placebokontrollierten randomisierten Studie vor (Worth $\mathrm{H}$ et al. Respiratory Research 2009; Epub ahead of print, 10: 69): 242 Patienten mit einer moderaten bis schweren COPD nahmen an dieser Untersuchung teil. Ihre Begleitmedikation bestand aus Beta-2Mimetika, Anticholinergika, inhalativen Kortikosteroiden oder Theophyllin. In der Studie erhielten sie zusätzlich dreimal täglich 200 mg Cineol oder Placebo.

Nach einer Therapiedauer von sechs Monaten während der Winterzeit war un- ter Cineol die Exazerbationrate mit 0,4 gegenüber 0,9 unter Placebo signifikant vermindert. Die Dauer der Exazerbationen war ebenfalls signifikant reduziert (4,5 vs. 5,7 Tage). Auch die durchschnittliche Schwere der Exazerbation - auf einer Skala von 1 bis 3 - lag mit 0,8 in der Verumgruppe niedriger als in der Placebogruppe mit 1,4. Klinisch relevante unerwünschte Ereignisse traten unter Cineol nicht auf. In diesem Zusammenhang betonte Prof. Dr. Michael Pfeifer, Klinik Donaustauf, dass mit der Anzahl schwerer Exazerbationen auch die Mortalität deutlich ansteigt.

Der oxidative Stress durch freie Sauerstoffradikale ist ein entscheidender $\mathrm{Pa}$ thomechanismus bei der COPD, so Prof. Dr. Uwe Jürgens, Bonn. Er verwies auf eigene Studien mit isolierten humanen Monozyten. Die Applikation von Formoterol oder Budesonid zeigte kaum bzw. keine antioxidative Wirkung. Die Gabe von Cineol allein oder zusätzlich zu For-

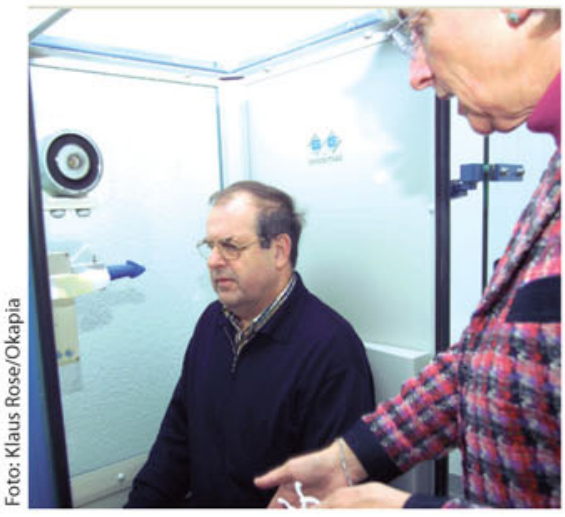

Häufige COPD-Exazerbationen verschlechtern die Prognose.

moterol und Budesonid minderte jedoch den oxidativen Stress deutlich.

$\diamond$ Dr. med. Andreas Fischer

Quelle: Pressekonferenz „Neue Therapieoption bei COPD: Soledum "Kapseln forte", München, 20. Oktober 2009 (Veranstalter: Casella-med)

\section{Mehr Lebensqualität für Schmerzpatienten}

_ Für die Behandlung starker Schmerzen stehen mit Opioiden sehr wirksame Substanzen zur Verfügung. Nicht selten sehen aber Ärzte wie Patienten den Einsatz im Hinblick auf die Nebenwirkungen kritisch. Mit der Fixkombination aus retardiertem Oxycodon und dem retardierten Opioidantagonisten Naloxon (Targin ${ }^{\odot}$ ) ist eine effektive Schmerzbekämpfung bei besserer Verträglichkeit möglich, denn die normale Darmfunktion bleibt erhalten.

In einer nicht interventionellen Multicenterstudie mit 7836 Schmerzpatienten wurden die Effizienz und Verträglichkeit des Kombinationspräparates dokumentiert. Die Patienten mit degenerativen Wirbelsäulenerkrankungen, Osteoporose oder Arthrose waren zu rund $75 \%$ mit Opioiden voreingestellt. Nach der Einstellung auf Oxycodon/Naloxon reduzierte sich die Schmerzintensität der Patienten um über $40 \%$ auf der Numerischen Ratingskala. Es zeigten sich auch eine Normalisierung der Darmfunktion sowie eine deutliche Anhebung der Lebensqualität.

\section{Tumorschmerzen mit} neuropathischer Komponente Bei Tumorpatienten, so Dr. Uwe Junker, Remscheid, können neben nozizeptiven Schmerzen oft auch neuropathische Schmerzen vorliegen. Auch dagegen hat sich die Fixkombination bewährt. Oxycodon hat eine hohe Affinität zu KappaOpioidrezeptoren, die bei neuropathischen Schmerzen hochreguliert sind.

Die gute Eignung von Oxycodon/Naloxon für Tumorschmerzpatienten auch mit neuropathischer Schmerzkomponente illustrierte Junker mit der Kasuistik einer 82-jährigen Patientin: Nach einer Chemotherapie wegen eines Kolonkarzi- noms entwickelte sie eine Zosterneuralgie und wurde stationär aufgenommen. Sie hatte starke brennende und elektrisierende Schmerzen vor allem im Nacken. Mit NRS 8 waren diese nachts stärker als am Tag mit NRS 5. Die Patientin litt deshalb unter Schlaflosigkeit. Wegen der hohen Schmerzintensität mit dominierender neuropathischer Komponente stellte Junker sie auf die Fixkombination ein. Zudem erhielt sie ein Pflaster mit 5\% Lidocain und ein Antikonvulsivum. Virustatisch behandelte er sie mit Brivudin. Nach zehn Tagen konnte die Frau mit einer Schmerzintensität von NRS 2 und normaler Darmfunktion entlassen werden. Außerdem schlief sie nachts wieder.

\footnotetext{
- Dr. med. Andreas Fischer

Quelle: Meet the Expert, Schmerzkongress,

Berlin, 8. Oktober 2009 (Veranstalter:

Mundipharma)
} 